\title{
Intra-Arterial Mechanical Thrombectomy: An Effective Treatment for Ischemic Stroke Caused by Endocarditis
}

\author{
Olafur Sveinsson $^{\mathrm{a}}$ Lars Herrman ${ }^{\mathrm{b}}$ Staffan Holmin ${ }^{\mathrm{b}}$ \\ ${ }^{a}$ Department of Neurology, Karolinska University Hospital, Stockholm, Sweden; \\ ${ }^{b}$ Department of Neuroradiology, Karolinska University Hospital, Stockholm, Sweden
}

\section{Keywords}

Infective endocarditis · Ischemic stroke $\cdot$ Middle cerebral artery occlusion $\cdot$ Intra-arterial mechanical thrombectomy

\begin{abstract}
Patients with stroke secondary to infectious endocarditis have a high in-hospital morbidity and mortality, with only one-third becoming functionally independent. Infective endocarditis is usually considered a relative contraindication to thrombolytic therapy. We describe 3 consecutive cases of acute middle cerebral artery occlusion due to infective endocarditis, who were all successfully treated with intra-arterial mechanical thrombectomy using the Solitaire device. From this limited experience, mechanical thrombectomy could be used as an effective acute treatment for ischemic stroke in patients with infective endocarditis. Mechanical thrombectomy is most likely a more effective and safer treatment than intravenous thrombolysis in this patient group.

(c) 2016 The Author(s) Published by S. Karger AG, Basel
\end{abstract}

\section{Introduction}

Patients with stroke secondary to infectious endocarditis have a high in-hospital morbidity and mortality, with only one-third becoming functionally independent [1]. Infective 


\section{Case Reports in Neurology}

Sveinsson et al.: Intra-Arterial Mechanical Thrombectomy: An Effective Treatment for Ischemic Stroke Caused by Endocarditis

endocarditis is usually considered a relative contraindication to thrombolytic therapy. This could be due to the following reasons: higher risk of hemorrhagic transformation of infarct, the possibility of intracranial mycotic aneurysms, and anticoagulant treatment in a subset of patients due to prosthetic valves. For these reasons treatment has consisted of supportive therapy and intravenous antibiotics. This treatment is obviously suboptimal, and improved treatment for this group of patients is much needed.

Here, we describe 3 consecutive cases of acute middle cerebral artery (MCA) occlusion due to infective endocarditis, who were all successfully treated with intra-arterial mechanical thrombectomy using the Solitaire device.

\section{Case 1}

A 33-year-old male with a prosthetic mitral valve, treated with warfarin, presented with high-grade $\left(40^{\circ} \mathrm{C}\right)$ fever. He was admitted to the infection clinic. Blood cultures where positive for Serratia marcescens. Transesophageal echocardiogram showed vegetation on the mitral valve, and treatment with meropenem and ciprofloxacin was initiated. At around midnight after 5 days of antibiotic treatment, the patient suddenly became aphasic and paretic on the right side (NIHSS 14). CT angiography of the brain showed a thrombus in the left M1 segment of the MCA (Fig. 1a). CT of the brain showed infarction of the left basal ganglia, but at the same time there was a large penumbra according to the mismatch between infarction size and perfusion disturbance (Fig. 1b). Since anticoagulation treatment and infectious endocarditis are relative contraindications to thrombolysis, no such therapy was administered. Instead, an endovascular procedure with mechanical thrombectomy was performed with a good outcome. Follow-up CT of the brain 1 day later showed infarction only of the left basal ganglia (Fig. 1c). The patient regained strength in the right side, but was left with mild dysphasia (NIHSS 1) at discharge. Three months later, the Modified Rankin Scale score was 1.

\section{Case 2}

A 67-year-old man with atrial flutter, hypertension, ischemic heart disease, and prosthetic mitral valve treated with warfarin and aspirin had gone to sleep at around 11 p.m. During the previous days he had had mild fever. He woke up at 4 a.m. with left-sided paresis. Forty minutes later, at the emergency department in another hospital, he had dysarthria, left homonymous hemianopsia, left central facial paralysis, reduced sensation in the left side of the body, and a paretic left arm, but could move the left leg a little (NIHSS 13). Initial CT of the brain showed no signs of infarction or hemorrhage. CT angiography showed an M1 occlusion on the right side (Fig. 2a). He was transferred to our hospital, where a repeat head CT showed a small infarction of the right basal ganglia (Fig. 2b), but at the same time CT perfusion revealed a large penumbra zone, according to infarct-perfusion deficit mismatch, in the right MCA territory (Fig. 2c). Mechanical thrombectomy was performed with a good functional outcome (NIHSS 3 at discharge).

At the same time he developed rising fever and elevated CRP $(156 \mathrm{mg} / \mathrm{L})$. Blood cultures were positive in 4/4 bottles for Enterococcus faecalis, and he was therefore treated with vancomycin and gentamicin that was changed to doctacycline and gentamicin 2 days later. Transesophageal echocardiogram showed a $3 \times 3 \mathrm{~mm}$ vegetation on the prosthetic mitral 


\section{Case Reports in Neurology}

valve. Because of the prosthetic valve it was decided to continue warfarin treatment. The patient received a 6-week course of antibiotics. A few months later NIHSS was 0, with only mild unsteadiness (Modified Rankin Scale score of 1).

\section{Case 3}

A 39-year-old woman with anorexia nervosa and staphylococcal sepsis suffered a cerebellar stroke a few days prior to presentation at the hospital. Examination showed a $1.5-\mathrm{cm}$ vegetation on a native mitral valve which was treated with cefotaxime. She suddenly became aphasic and had complete paresis of the right side (NIHSS 15). CT angiography showed a short occlusion of an M2 branch on the left side (Fig. 3a), and CT of the brain showed a manifest infarction in the frontal operculum (Fig. 3b) with a relatively large penumbra based on infarct-perfusion deficit mismatch (Fig. 3c). Thrombolysis was contraindicated, not the least because of the cerebellar infarction a few days earlier. Mechanical thrombectomy was performed successfully. At the time of discharge she had NIHSS 4 and the mitral vegetation had disappeared. She was subsequently treated for 4 weeks with cefotaxime. At 3 months after ictus her Modified Rankin Scale score was 2.

\section{Discussion}

Intravenous thrombolysis is usually considered a relative contraindication in patients with infective endocarditis. When used, it has shown mixed results, including reports of an increased risk of intracerebral hemorrhage. In a series of 8 patients, 5 suffered from symptomatic intracerebral hemorrhage after thrombolysis [2,3]. Until now there have only been case reports of mechanical thrombectomy with relatively good outcomes. Out of 7 cases, there have been no hemorrhages, and the clinical outcome has been good except in 2 cases [4]. In addition, thrombectomy has been confirmed in recent randomized trials to be a very effective treatment in patients with ischemic stroke due to large artery occlusions of the anterior circulation [5-9]. Since ischemic stroke due to infective endocarditis is relatively rare, randomized trials are not possible.

All our cases made a good recovery. From this limited experience, mechanical thrombectomy could be used as an effective acute treatment for ischemic stroke in patients with infective endocarditis. Even though mechanical thrombectomy has been proven to be an effective acute treatment in ischemic stroke, there are only a few case reports on mechanical thrombectomy in stroke caused by endocarditis. As a proof of principle, it is important to show that the method is safe and effective. Additionally, the importance of mechanical thrombectomy is even greater due to the fact that thrombolysis is contraindicated in patients with stroke caused by endocarditis.

\section{Statement of Ethics}

All 3 patients provided informed consent for their participation in the case series. 


\section{Case Reports in Neurology}

\section{Disclosure Statement}

The authors have nothing to disclose. This case series was not funded.

\section{References}

1 Sonneville R, Mirabel M, Hajage D, et al: Neurologic complications and outcomes of infective endocarditis in critically ill patients: the ENDOcardite en REAnimation prospective multicenter study. Crit Care Med 2011;39:1474-1481.

-2 Ong E, Mechtouff L, Bernard E, et al: Thrombolysis for stroke caused by infective endocarditis: an illustrative case and review of the literature. J Neurol 2013;260:1339-1342.

-3 Bhuva P, Kuo SH, Claude Hemphill J, Lopez GA: Intracranial hemorrhage following thrombolytic use for stroke caused by infective endocarditis. Neurocrit Care 2010;12:79-82.

4 Kim JM, Jeons JS, Kim YW, Kang DH, Hwang YH, Kim YS: Forced arterial suction thrombectomy of septic embolic middle cerebral artery occlusion due to infective endocarditis: an illustrative case and review of the literature. Neurointervention 2014;9:101-105.

5 Berkhemer OA, Fransen PS, Beumer D, et al: A randomized trial of intraarterial treatment for acute ischemic stroke. N Engl J Med 2015;372:11-20.

6 Goyal M, Demchuk AM, Menon BK, et al: Randomized assessment of rapid endovascular treatment of ischemic stroke. N Engl J Med 2015;372:1019-1030.

7 Campbell BC, Mitchell PJ, Kleinig TJ, et al: Endovascular therapy for ischemic stroke with perfusionimaging selection. N Engl J Med 2015;372:1009-1018.

8 Saver JL, Goyal M, Bonafe A, et al: Stent-retriever thrombectomy after intravenous t-PA vs. t-PA alone in stroke. N Engl J Med 2015;372:2285-2295.

\$ Jovin TG, Chamorro A, Cobo E, et al: Thrombectomy within 8 hours after symptom onset in ischemic stroke. N Engl J Med 2015;372:2296-2306.
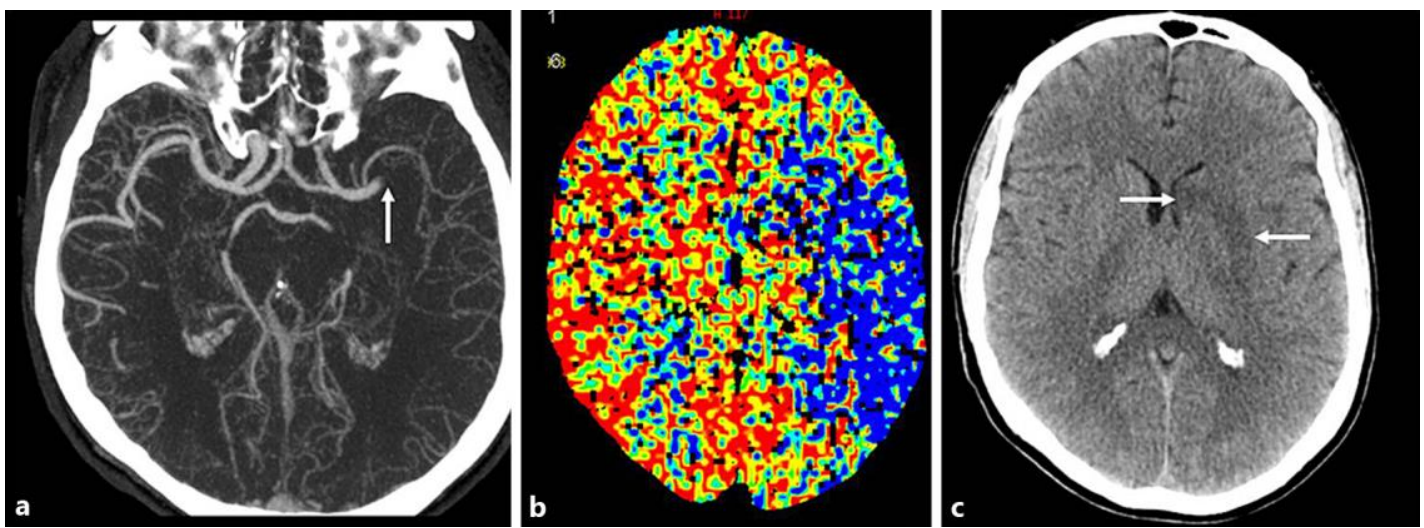

Fig. 1. a CT angiography of the brain showed an occlusion in the distal part of the left M1 segment of the middle cerebral artery (arrow). b CT perfusion of the brain demonstrated increased mean transit time in the middle cerebral artery territory on the left side (blue area) as compared to the right side. c CT of the brain without intravenous contrast displayed infarction of the basal ganglia on the left side with decreased attenuation of the caudate (upper arrow) and lentiform nucleus (lower arrow). 


\section{Case Reports in Neurology}

\begin{tabular}{l|l}
\hline Case Rep Neurol 2016:8:229-233 \\
\hline DOI: 10.1159/000452213 & $\begin{array}{l}\text { @ 2016 The Author(s). Published by S. Karger AG, Basel } \\
\text { www.karger.com/crn }\end{array}$ \\
\hline
\end{tabular}

Sveinsson et al.: Intra-Arterial Mechanical Thrombectomy: An Effective Treatment for Ischemic Stroke Caused by Endocarditis
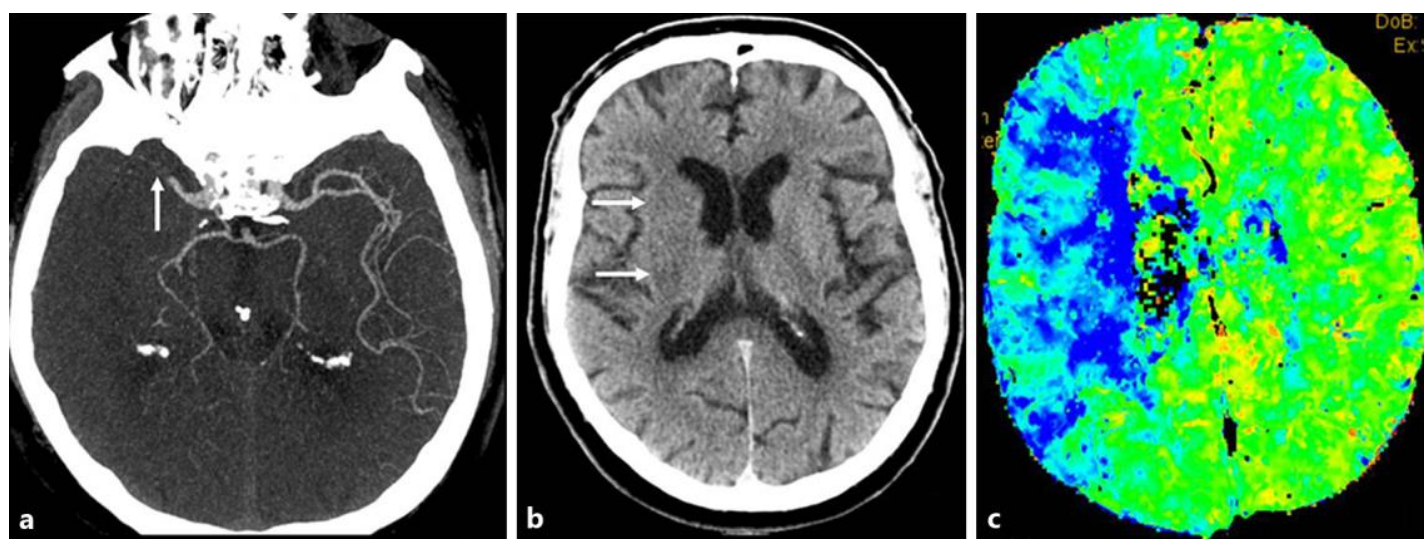

Fig. 2. a CT angiography of the brain showed occlusion of the M1 segment of the middle cerebral artery on the right side (arrow). b CT of the brain exhibited decreased attenuation of the right basal ganglia in keeping with infarction (arrows). c CT perfusion of the brain showing increased mean transit time in the middle cerebral artery territory on the right side (blue area).
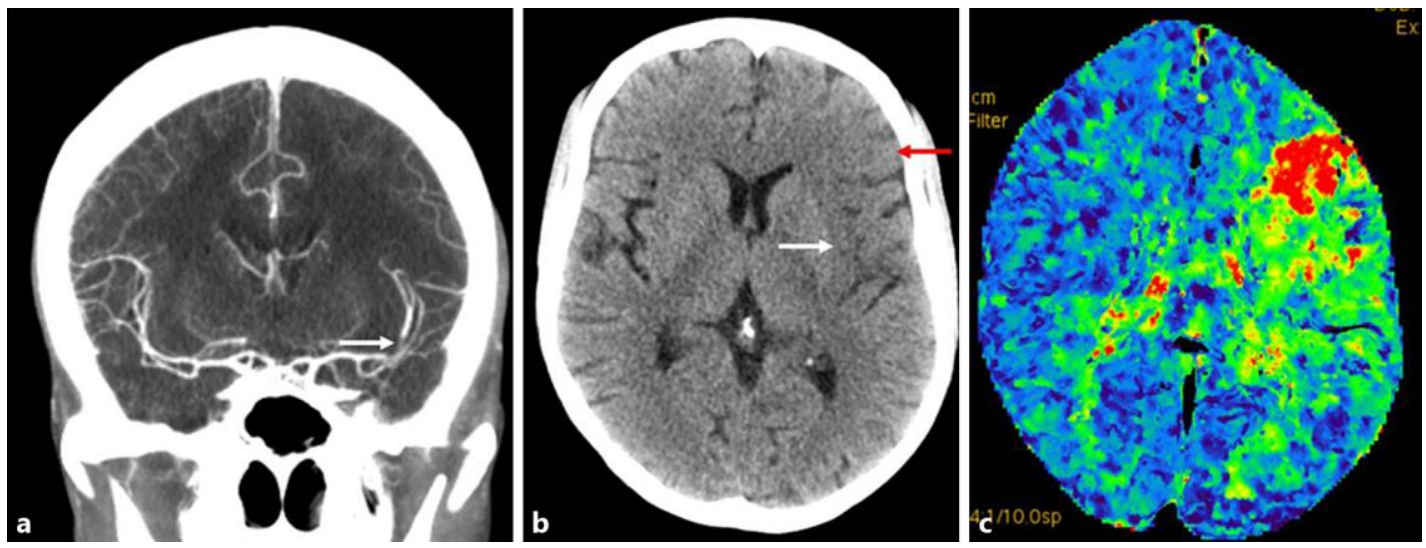

Fig. 3. a Coronal view. CT head angiography revealed a contrast-filling defect in the MCA bifurcation on the left side representing a thrombus (arrow). b CT scan of the brain showed decreased attenuation in the frontal operculum (red arrow) and insula (white arrow) on the left side compatible with infarction. c CT brain perfusion with increased mean transit time in the area of the left MCA territory. The red and green area defines the perfusion deficit. 